Article

\title{
Environmental Consciousness in Daily Activities Measured by Negative Prompts
}

\author{
Ai Hiramatsu *, Kiyo Kurisu and Keisuke Hanaki \\ Received: 30 September 2015; Accepted: 23 December 2015; Published: 26 December 2015 \\ Academic Editors: Marc A. Rosen, Yasuhiro Fukushima, Yasushi Kondo, Shinsuke Murakami, \\ Masaharu Motoshita and Matthias Finkbeiner \\ Department of Urban Engineering, The University of Tokyo, 7-3-1 Hongo, Bunkyo-ku, Tokyo 113-8656, Japan; \\ kiyo@env.t.u-tokyo.ac.jp (K.K.); hanaki@env.t.u-tokyo.ac.jp (K.H.) \\ * Correspondence: ai@env.t.u-tokyo.ac.jp; Tel.: +81-3-5841-6248; Fax: +81-3-5841-8530
}

\begin{abstract}
The gap between people's attitude and action as regards environmental issues has been pointed out even while surveys registered an increase in people's environmental awareness. Among the possible reasons is that people tend to automatically answer "yes", as most surveys on environmental consciousness use positively-phrased questions or prompts. To remove the "yes-bias" in previous surveys, this present study conducted in Japan a large-scale questionnaire survey on environmental consciousness using negative prompts and free-answered prompts on which behaviors people feel good/bad/uncertain for the environment. This study also investigated peoples' psychological factors and concrete pro-environmental behaviors (PEBs) in daily life. The results of the questionnaire with negative prompts showed that the rate of people's consciousness to the environment was lower compared with other surveys. Through factor analysis, five psychological factors were extracted as the explanatory factors of environmental attitude. Demographic effects on the consciousness and PEBs were also observed. Comparison of free-answers on concrete daily behaviors among five different environmentally conscious groups showed there were certain phases in the perception of PEBs based on consciousness level. Similar common behaviors were highly ranked as both PEB and doubtful behaviors, indicating that people were worried about actions that involve a trade-off relationship from diversified standpoints.
\end{abstract}

Keywords: pro-environmental behavior (PEB); psychological factor; yes-bias tendency; attitude-behavior gap; questionnaire survey; factor analysis; cluster analysis; text analysis

\section{Introduction}

Environmental awareness in Japan has increased and became distinguished throughout the world, alongside environmentally-conscious European nations such as Sweden and Germany [1]. Environmental education for citizens has been actively implemented by various organizations following efforts by both the government and industry associations to set stricter environmental standards since the end of the 1980s. Since the 1990s, industry associations have applied economic incentives to address environmental pollution as well as the global environmental problems. However, while environmental awareness has improved in recent years, especially after the earthquake in 2011 in Japan, a gap between people's attitude and action as regards environmental issues has been pointed out. People's actions do not reflect such high levels of environmental consciousness. Such contradiction between attitude and action has been mentioned in previous studies [2].

Most surveys on environmental consciousness conducted by national or local governments have used positively phrased questions or prompts. The attitude-behavior gap could be attributed to the positive questions or prompts in the questionnaire; the overestimation of environmental awareness might have induced this gap. Among the reasons for this gap is the "acquiescence bias" or "yes-bias" 
tendency, which refers to the tendency of respondents to choose a certain response category regardless of the item's content [3,4]. People tend to unconsciously or automatically say "yes" when responding to a questionnaire to meet certain expected societal values or feel a sense of moral or social value. Hence, under a positively phrased questionnaire, people might have unconsciously pretended to be concerned when asked whether they are conscious about the environment even though they are less concerned. Chao et al. [5] reported that there was a certain difference between self and other reporting in answering environmental behavior; he advised researchers to be careful in interpreting results of only self-reported behavior as these results are usually higher than the actual turnout. Kaiiser et al. [6] and Milfont [7] assumed that social desirability is among the factors explaining the high attitude-behavior gap. They concluded that a socially-desirable response had only a weak direct effect on environmental attitudes and self-reported behaviors.

To further understand the cause of the attitude-behavior gap in this regard, a large-scale questionnaire survey using negative prompts was conducted in our research to investigate the effect of positive prompts. This survey was conducted to obtain results that were free from any "yes-bias" tendencies present in the past surveys. The authors also examined the characteristics of respondents.

In recent years, the relationship among psychological variables that influence environmental attitude, which, in turn, leads to pro-environment behavior PEB, has been studied. The theories of planned behavior [8] and value belief norm [9] were famous as pioneering models that explain people's behavior. These theories include elements such as psychological factors of values, normative beliefs, perceived behavioral control, and intention, among others. Various environmental psychological models have been developed and improved from applying and expanding the above models using new other factors such as altruism [10]. PEBs are very complicated and cannot be easily divided into positive or negative positions. However, this research does not discuss the interrelation of psychological variables and model development but rather focuses on the style of expression of statements in questionnaires.

This research also aims to understand people's perceptions of appropriate behaviors towards the environment in daily life. Such perceptions presumptively affect the processing of information from consciousness to attitude and, finally, to a realized action. The survey contained prompts as regards behaviors such as whether people perceived certain behaviors as good or bad for the environment. Analyzing and classifying answers based on the level of respondents' consciousness, and concrete PEBs will help identify barriers to reaching a realized action as well as analyze psychological factors affecting the processing of information. The results of the study will assist in the design of broader and more effective countermeasures and environmental education.

\section{Methods}

\subsection{Questionnaire Design}

The questionnaire survey on people's environmental consciousness in daily activities was designed with consideration of the "yes-bias" tendency and the fact that people display PEBs without real consciousness of the environment. As such, 22 of 25 prompts on environmental awareness in daily behaviors were asked in negative ways (Table 1) using the six-point Likert Scale (strongly, mostly, slightly agree/disagree). Prompts were set from various standpoints based on the items used for measuring the level of environmental consciousness in governments and other organizations. Prompts asked how much people emphasize environmental issues, who is responsible for the environmental problems, how people perceive the extent of influence of their behaviors, how people balance PEBs with convenience and cost (economic efficiency), and what are the environmental information available in society. Prompts were also selected based on the objective to compare the present study with existing surveys on consciousness. As such, a number of them were displayed as reversed prompts of existing surveys. 
Table 1. List of negative prompts.

\begin{tabular}{|c|c|}
\hline No. & Prompt \\
\hline Q1_1 & $\begin{array}{l}\text { My Pro-Environment Behaviors (PEBs) are based on custom rather than } \\
\text { environmental consciousness. }\end{array}$ \\
\hline Q1_2 & My concern for environmental issues is not particularly high. \\
\hline Q1_3 & I do not know how much media information on the environment is reliable. \\
\hline Q1_4R & Environmental issues are important problems to be solved. \\
\hline Q1_5 & In reality, I do not particularly care about the environment. \\
\hline Q1_6 & I cannot understand a person who is enthusiastic about environmental activities. \\
\hline Q1_7 & The terms "eco-/environmentally friendly" are suspicious. \\
\hline Q1_8 & $\begin{array}{l}\text { Companies and industries should take more efforts than individuals to improve } \\
\text { the environment. }\end{array}$ \\
\hline Q1_9 & Individual action will not improve the environment. \\
\hline Q1_10 & I do not think my behavior is responsible for global warming. \\
\hline Q1_11 & I do not have enough time to actively change the environment. \\
\hline Q1_12 & $\begin{array}{l}\text { When purchasing (e.g., energy, resources, waste), I do not particularly care about } \\
\text { the environment. }\end{array}$ \\
\hline Q1_13R & PEBs should be adopted even if they are bothersome. \\
\hline Q1_14 & I will not display PEBs when I do not have the attention of others or society. \\
\hline Q1_15 & $\begin{array}{l}\text { Even if they are good for the environment, I avoid PEBs because I do not want to } \\
\text { be inconvenienced. }\end{array}$ \\
\hline Q1_16 & I display PEBs to save money. \\
\hline Q1_17R & Individual action is important to solve environmental problems. \\
\hline Q1_18 & There is not enough useful information on adopting PEBs. \\
\hline Q1_19 & Honestly, I do not know what behaviors are good or bad for the environment. \\
\hline Q1_20 & The solution to global warming will come about independently from me. \\
\hline Q1_21 & An individual inaction will not significantly influence the environment. \\
\hline Q1_22 & For me, there are more important things than environmental issues. \\
\hline Q1_23 & I display PEBs depending on whether others are paying attention to my behaviors. \\
\hline Q1_24 & I became fed up with being told to display PEBs. \\
\hline Q1_25 & $\begin{array}{l}\text { I do not care about environmental issues even if they were to destroy the earth in } \\
\text { the future. }\end{array}$ \\
\hline
\end{tabular}

R: reverse prompt.

The survey included free-answer prompts. These prompts cover questions on behaviors the respondents usually feel are good for the environment $(\mathrm{Q} 2)$, those they are uncertain of as to whether they are good or bad for the environment (Q3), and those they generally consider as good for the environment even while they do not agree (Q4). No character limit was set and the respondent could enter up to three answers per free prompt. The survey also contained questions on socio-demographics (e.g., age, gender, occupation, resident area, and household income).

The survey was conducted online among Japanese aged 15-69 from 24 January to 27 January, 2014 through a research marketing company. People who registered as monitors of the company throughout Japan answered the questionnaire voluntarily, obtaining points in return. A total of 72,738 valid responses (response rate: $41.8 \%$ ) were collected in accordance with the ratio of the population in their respective areas of residence, gender categories, and age groups. 


\subsection{Questionnaire Survey}

Various methods are used in social research surveys such as mailing, telephone, and online surveys as well as face-to-face interviews. This present study utilized an online survey, which has been an increasingly popular method since the rapid growth of the Internet. Among the advantages of this method is that it allows a large-scale questionnaire survey to be conducted in a short period. The rate of response also tends to be higher compared with other methods. Further, respondent distribution can be easily adjusted so that this method is suitable for nationwide investigation, except for areas where the Internet is not readily accessible. Although Internet is widely available in Japan, older people are less likely access the Internet. As such, this present study targeted the younger demographic (aged 15-69) so that online survey can be applied to get enough samples.

The authors removed the answers in the following cases during the initial response collection to address contradicting responses: answer time was too short; answers were unrelated; inappropriate values and words were entered; and the entered attribute was inconsistent with registered information. The authors also checked the responses bearing the same values in all items that were less than $0.2 \%$ and those that had inconsistent values with reversed prompts equal to $1.5 \%$. As it was difficult to identify clearly contradictory responses, the answers were retained. Further, the overall trend did not change in either case.

\subsection{Analysis}

To analyze the responses to negative prompts, the values of a six-point Likert Scale were set as follows: strongly agree $=6$; mostly agree $=5$; slightly agree $=4$; slightly disagree $=3$; mostly disagree $=2$; and strongly disagree $=1$. The assignment of values indicated that the larger the value of the response, the more negative the respondent's attitude is to the environment, except for cases of reverse prompts. The difference in the people's responses was examined by comparing them with the existing surveys.

SPSS Ver. 22 (IBM Co., New York, NY, USA) was utilized for factor and cluster analyses. Factor analysis was conducted applying the maximum-likelihood method and promax-rotation to extract characteristic psychological factors that explain people's environmental consciousness. The extract factors were positioned as "sub-scales." Each sub-scale score was calculated as the mean of appropriate prompts. How each sub-scale score differs in the population was also investigated.

The cluster analysis was conducted based on factor scores obtained from the factor analysis. The respondents were categorized into clusters according to their level of environmental consciousness. Because the sample size was too large to create a dendrogram, the number of clusters was assumed and analyzed. Exchanging the number of clusters, analysis was conducted repeatedly to find the appropriate number of clusters.

Meanwhile, text analysis was conducted to analyze free-answer responses using the software Text Mining Studio 5.1 (NTT DATA Mathematical Systems Inc., Tokyo, Japan). First, sentences were decomposed into morphemes and the fluctuations of descriptions were modified. Then, the synonymous words were combined into one word. If the same word was used multiple times in one answer, the number of occurrences was counted as one.

Word-frequency analysis was applied to detect the most frequently used phrases and words to reveal major topics among respondents. Through the extraction of feature words, the level of people's consciousness and concrete behaviors were connected and investigated. The complementary similarity measure, which counted words and considered the size of population of the group, was applied when the appearance frequency was compared among socio-demographics or clusters. Co-occurrence network analysis was used to explore words with similar appearance patterns as well as determine the strength of co-occurrences and interrelationship between words. In this present study, frequently co-occurring words with "use" and "not use" were investigated. 


\section{Results}

\subsection{Respondents' Distribution}

The socio-demographics of respondents were summarized in Supplementary Materials (Tables S1-S6). Census data on age, gender, and regions were also shown [11]. While the proportion of population was adjusted on prefecture level, the table showed regional data to avoid complication. All ages and gender were almost equally represented except for teens (3.4\%). Company employees accounted for one third of the total respondents. People who stay at home or work part time also accounted for a third. The unemployment rate including retired people was $9.6 \%$ and self-employed, professionals, or government employee workers comprised $13.7 \%$. Of the total respondents, $15.1 \%$ live alone and $73.7 \%$ live either with a family of two to four people or share a house with others. As for income, the rate of household income after taxes under 2 million yen was $10.6 \%$, and the rate of $2-4,4-6$, and 6-8 million yen accounted for $21.5 \%, 22.1 \%$, and $14.3 \%$, respectively. The Kanto region comprised the largest population (34.8\%). It was followed by the Kansai region (17.7\%) and the Chubu region $(16.8 \%)$.

\subsection{Results of Responses to Negative Prompts}

\subsubsection{People's Environmental Consciousness}

Figure 1 shows the results of people's environmental consciousness. Eighty percent of the respondents agreed with prompt numbers Q1_1, Q1_3, and Q1_16, whereas 60\%-70\% of people responded "agree" to prompt numbers Q1_8, Q1_15, Q1_18, Q1_19, and Q1_22. Many people considered other matters other than the environment and did not have enough reliable information on PEB. Moreover, displaying PEB depended on custom, economic benefit, and convenience. Meanwhile, 70\% answered "disagree" to prompt numbers Q1_10, Q1_14, Q1_21, and Q1_23. The respondents' disagreements with these prompts confirmed the perception that individual efforts and their effects are important. People's positive attitudes to the environment were observed based on these results.

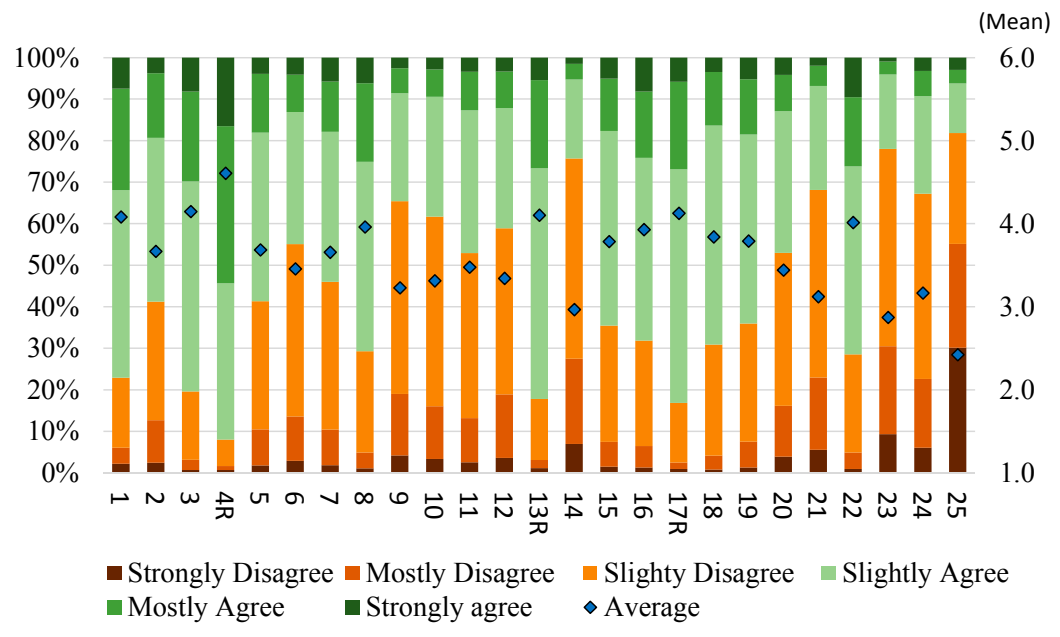

Figure 1. People's environmental consciousness in daily life.

The results illustrated in Figure 2 showed that women were more environmentally conscious than men. However, women displayed PEBs based on customs or to save money rather than on environmental consciousness. Compared with men, women were more likely to perceive having a lack of useful information on environmental issues. The results also indicated that elderly people generally tended to be more environmentally conscious than younger people (Figure 3). However, people in their $20 \mathrm{~s}$ had the least positive attitude. Responses to Q1_15, Q1_16, and Q1_25 varied largely depending 
on generation. Although people agreed that they wanted to avoid inconvenience and displayed PEB to save money as well as disagreed that they did not mind the possibility of earth's extinction, the level of answers was different. People aged above 30 considered the impact of their purchase on environment, whereas people aged below 20 did not (Q1_12). Meanwhile, all generation had similar answers on Q1_8 and Q1_10 but slightly agreed that companies should make greater efforts to environmental change and slightly disagreed that they were not responsible for the environment. In some instances, special situations were especially seen in people in their 60s; for example, certain respondents in the group dedicated the most time and placed high priority on environmental problems.

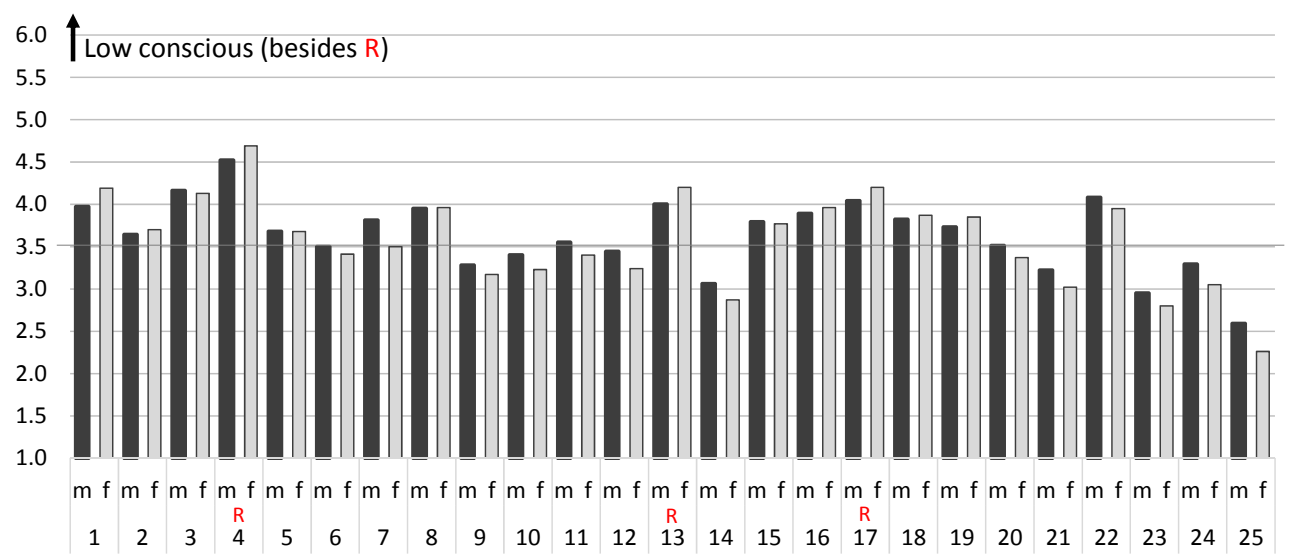

Figure 2. Average of responses by gender (m: male, f: female).

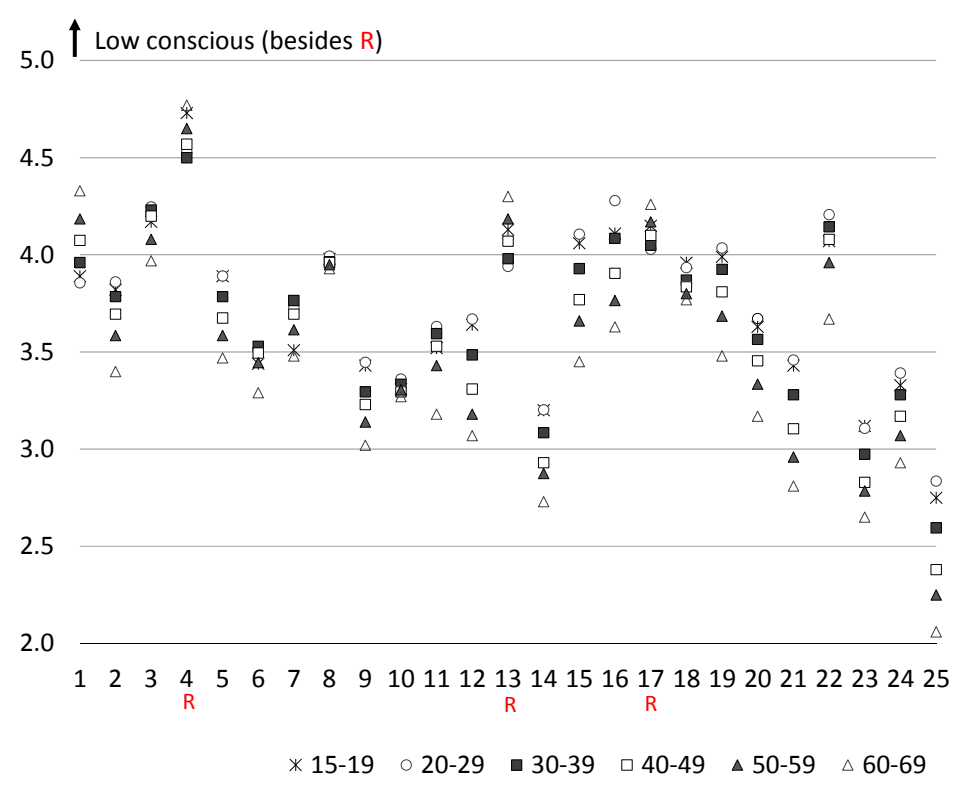

Figure 3. Average of responses by age.

\subsubsection{Psychological Factors of Environment Based on Factor Analysis}

To detect the psychological elements and classify the respondents, factor analysis was conducted. Prompts whereby factor scores fell under 0.4 (Q1_11, Q15, Q24, and Q25) were removed after establishing all prompts. Five factors (sub-scales), A-E, were extracted as shown in Table 2 to explain $60 \%$ of people's environmental consciousness. Factor correlation matrix is shown in Table 3. 
Table 2. Results of factor analysis.

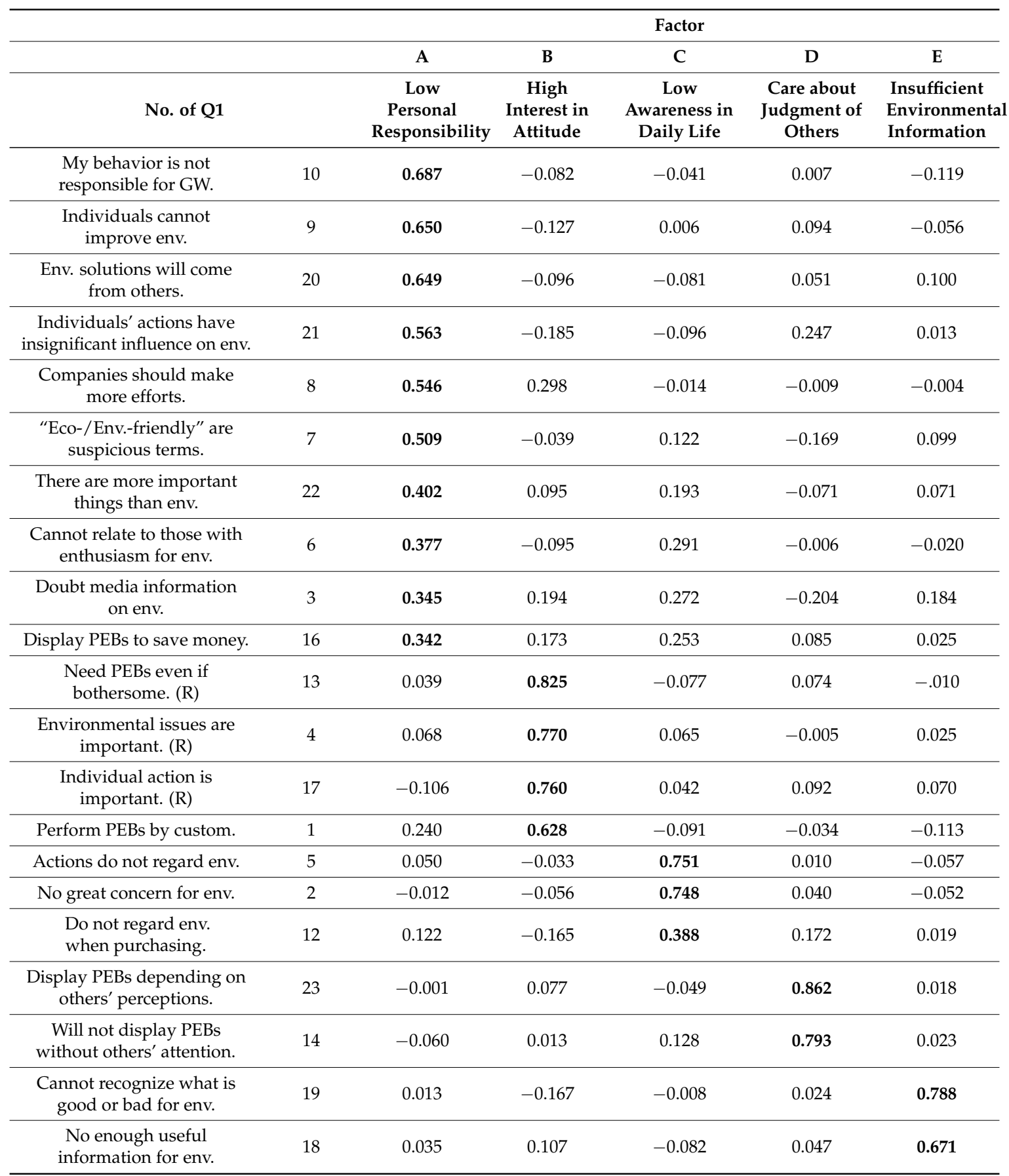

Bold: Extracted factor (Maximum-likelihood method, Promax-rotation); GW: global warming, env.: environment.

Table 3. Factor correlation matrix.

\begin{tabular}{cccccc}
\hline Factor & A & B & C & D & E \\
\hline A & 1.000 & -0.521 & 0.668 & 0.612 & 0.481 \\
B & -0.521 & 1.000 & -0.424 & -0.606 & 0.001 \\
C & 0.668 & -0.424 & 1.000 & 0.452 & 0.450 \\
D & 0.612 & -0.606 & 0.452 & 1.000 & 0.238 \\
E & 0.481 & 0.001 & 0.450 & 0.238 & 1.000 \\
\hline
\end{tabular}


Factor "A" represents the psychological factor showing low responsibility awareness of oneself, including the following prompts: "My behavior is not responsible for global warming"; "Individual action will not improve the environment"; and "Companies and industries should initiate more efforts toward environmental change than individuals".

Factor " $\mathrm{B}$ " regards the psychological factor having a high interest in environmental problems and included mainly reverse prompts such as "We should perform PEBs even if they are bothersome" and "Environmental issues are important".

Factor " $\mathrm{C}$ " represents the psychological factor having low environmental awareness in daily life. People with a high " $\mathrm{C}$ " score did not care about the environment when purchasing or taking various actions in their daily lives.

Factor " $\mathrm{D}$ " regards the psychological factor showing awareness of other's judgments or perceptions of others. People with a high " $\mathrm{D}$ " score performed PEBs depending on whether they feel they are being observed by other individuals or society as a whole.

Factor " $E$ " represents the psychological factor sensing information shortage, including prompts such as "I do not know what behaviors are good or bad for the environment" and "There is not enough useful information on adopting PEBs".

The sub-scale score was calculated as a mean of related prompts. Each sub-scale's mean and standard deviation are shown in Table 4. On average, people had slight personal responsibility and slightly high environmental attitude and awareness in daily life. On average, too, they cared less about other's judgment and slightly felt there was insufficient environmental information.

Table 4. Descriptive statistics of sub-scale scores.

\begin{tabular}{cccc}
\hline & Mean & Standard Deviation & Number of Prompts \\
\hline A & 3.63 & 0.64 & 10 \\
B & 4.23 & 0.71 & 4 \\
C & 3.56 & 0.86 & 3 \\
D & 2.92 & 0.89 & 2 \\
E & 3.81 & 0.79 & 2 \\
\hline
\end{tabular}

Difference from mean of each sub-scale by socio-demographic was also calculated and shown in Tables S7-S12 of Supplementary Materials. Sub-scale scores of A, C, and D of male became higher than those of female and sub-scale scores of $B$ and $E$ became lower than those of female. This indicated that males demonstrated lower personal responsibility and lower environmental attitude both in general and in daily life. Compared with women, men cared more about the perceptions of others and were more knowledgeable on the environment.

A correlation between factors and age/income was found. Age/income were negatively correlated with $\mathrm{A}, \mathrm{C}, \mathrm{D}$ and $\mathrm{E}$ and positively correlated with $\mathrm{B}$. The older the people get and the higher the income they earn, the more they feel responsible for the environment. They also had higher awareness and possessed more information.

As for occupation, students had much lower responsibility and awareness in daily life than others even while they expressed interest in environmental issues. They cared about the perception of others and felt they had a lack of information on the environment. Meanwhile, stay-at-home people demonstrated higher responsibility and awareness for the environment and did not care about the perceptions of others. Company employees demonstrated generally lower attitude for the environment in general, whereas professionals showed higher environmental interest. Professionals, government employees, self-employed people, and unemployed (retired) ones also demonstrated higher awareness in daily life and possessed more information. They were also unaffected by other's judgments.

Contrary to expectations, no particular difference was found among regions. The number of people in the household was a unique feature among the factors. People who live alone tended to demonstrate lower awareness and responsibility as well as performed PEB only with consideration 
of the perception of others or society. Meanwhile, people who live with other people showed higher concern and personal responsibility and displayed PEB regardless of others' perceptions. However, people living with more than two others showed almost no difference to the mean.

\subsubsection{Respondent Categories Based on Environmental Consciousness by Cluster Analysis}

The cluster analysis was conducted to classify the respondents and identify their characteristics. Scores on Factor B were reversed to display the negative consciousness as a positive value. The meaning of each factor score was explained in Table 5, whereas Figure 4 showed the results of five clusters in which the characteristics are clearly indicated.

Table 5. Meaning of the factor score.

\begin{tabular}{|c|c|c|c|c|}
\hline & Factor & High Score & $\leftrightarrow$ & Low Score \\
\hline A & Personal responsibility & $\begin{array}{l}\text { Do not feel responsibility } \\
\text { of individual behavior } \\
\text { for the environment }\end{array}$ & $\leftrightarrow$ & $\begin{array}{c}\text { Emphasize the responsibility } \\
\text { of individual behavior for } \\
\text { the environment }\end{array}$ \\
\hline B & $\begin{array}{l}\text { Attitude towards } \\
\text { environment }\end{array}$ & $\begin{array}{c}\underline{\text { Low interest in }} \\
\text { environmental issues }\end{array}$ & $\leftrightarrow$ & $\begin{array}{l}\text { High interest in } \\
\text { environmental issues }\end{array}$ \\
\hline $\mathrm{C}$ & $\begin{array}{c}\text { Environmental } \\
\text { awareness in daily life }\end{array}$ & Low awareness & $\leftrightarrow$ & High awareness \\
\hline $\mathrm{D}$ & $\begin{array}{l}\text { Caring about } \\
\text { perceptions of others }\end{array}$ & $\begin{array}{l}\text { Act if being observed by } \\
\text { others and society }\end{array}$ & $\leftrightarrow$ & $\begin{array}{l}\text { Act regardless of attention } \\
\text { from others or society }\end{array}$ \\
\hline E & $\begin{array}{l}\text { Environmental } \\
\text { information }\end{array}$ & $\begin{array}{l}\text { Information is } \\
\text { insufficient }\end{array}$ & $\leftrightarrow$ & Information is enough \\
\hline
\end{tabular}

The authors put the underline to prevent misunderstanding because the score of factor B was reversed. " $\leftrightarrow$ " (Left right arrow) means there is a score range between the high score and the low score.

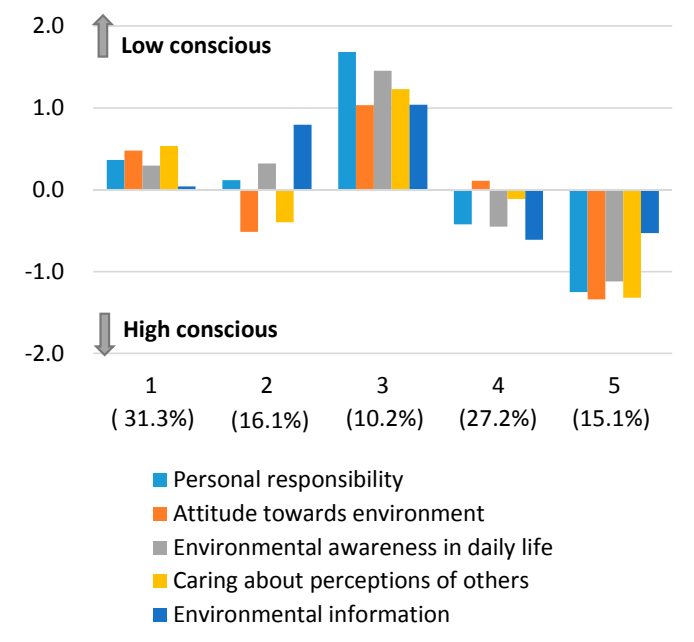

Figure 4. Results of cluster analysis (five clusters).

Cluster 1 includes a middle group that showed slightly low environmental consciousness. This group showed a low interest in the environment and low consciousness in daily life. People in this group felt minor personal responsibility for the environment, and did not engage in PEBs when others were not observing them.

Cluster 2 is made up of a group of people sensing minor personal responsibility in everyday life. However, they showed interest in the environment in general. They felt that there was a lack of environmental information. This group also had a significantly high proportion of women in their $20 \mathrm{~s}$ through $40 \mathrm{~s}$. 
Cluster 3 is made up of those who were the least environmentally conscious and there is a high proportion of young men. This group consistently displayed a low environmental attitude, did not act without others' attention, and lacked information on the environment.

Cluster 4 is comprised of a middle group that displayed a slightly high environmental consciousness. This group felt a certain personal responsibility to some extent, had more environmental consciousness in daily life, and exhibited PEBs regardless of others' attention. They also perceived that there was sufficient information on environmental issues.

Cluster 5 includes the most environmentally conscious group, which had a high proportion of elderly people, especially women. This group had a strong consciousness about the environment, felt a strong sense of individual responsibility, and usually performed PEBs on a daily basis regardless of others' attention. Although this group already received environmental information to some extent, it was indicated that they demanded more extensive information by the comparatively lower score on information factor.

The results showed only a minor regional difference. Kanto, Kyushu, and Okinawa had slightly higher ratios of clusters 4 and 5, which were higher conscious groups. As for the cluster percentage by gender, middle groups (clusters 1 and 4 ) accounted for the majority ( $57 \%$ women, $60 \%$ men). Among women, cluster 3 (the low conscious group) accounted for $7.6 \%$ and cluster 5 (the very conscious group), $16.6 \%$ (details were shown in Supplementary Materials of Figure S1). Among men, the ratio of clusters 3 and 5 were almost the same at $12.8 \%$ and $13.7 \%$, respectively. Although cluster 1 was the largest proportion cluster for age groups leading up to $40 \mathrm{~s}$, this changed to cluster 4 for age groups over 50. Such results indicate that environmental awareness increased with age. The proportion of cluster 3 in people in their $20 \mathrm{~s}$ was the highest among all generations. This age range had the greatest percentage of the lower-conscious cluster, indicating the least conscious generation. In contrast, the higher conscious clusters were included in the $60 \mathrm{~s}$ age range. As for the proportion of cluster by household income, environmental consciousness also increased proportionally with annual income. In the group earning 2 million yen or more, although the proportion of cluster 3 maintained the similar level as $10 \%$, the proportion of clusters increased with greater environmental consciousness and higher rates of displaying PEB in everyday life. The rates of the clusters with high environmental consciousness were outstanding, especially in the income group earning more than 15 million yen.

More subdivided cluster analyses were also conducted as the number of samples was quite large and the characteristics were rather generalized. The results of 20 clusters were shown in Figure S2. Respondents summarized in above five clusters were divided into more groups according to the level of environmental awareness; the distinctive groups were also extracted. The characteristic groups are described as follows. A group that contained more men perceived that there was enough information on environmental issues and showed very low consciousness (cluster 3 in Figure S2). There was also a group with low personal responsibility, low consciousness, and that disregard others' perceptions of their environmental actions (cluster 7 in Figure S2). Another group had limited knowledge of environmental issues and barely made any individual effort, although they showed interest in the environment (cluster 11 in Figure S2). Certain groups included a high proportion of women and were made up of those who perceived a lack of information even though they showed high interest in the environment (clusters 6 and 12 in Figure S2). A group did not consider environmental problems to be very important but had knowledge on environmental issues and personally acted on their environmental consciousness in daily life to some extent (cluster 14 in Figure S2).

\subsection{Results of Answers to Free-Answer Prompts}

\subsubsection{Extraction of Behaviors Based on Respondents' Recognition by Text Analysis}

Text analysis was conducted for free-answers to prompts Q2 (behaviors you usually feel are good for the environment), Q3 (behaviors that you are uncertain of as to whether they are good or bad for the environment), and Q4 (behaviors that are generally thought to be good for the environment 
though you do not agree). Although no limitation on letter count was set, majority of respondents answered at the word level. Among 72,738 respondents, 69,707 people had certain input to Q2, whereas 66,705 people responded to Q3 and 62,361 people to Q4.

The top 20 behaviors obtained by word frequency analysis in Q2, Q3, and Q4 were shown in Table 6. The results showed that similar common behaviors were highly ranked in all prompts, including garbage separation, power saving, recycling, and eco bags.

Table 6. Top 20 frequently answered behaviors asked by prompts Q2-Q4.

\begin{tabular}{|c|c|c|c|c|c|c|}
\hline & \multicolumn{2}{|c|}{$\begin{array}{l}\text { (Q2) Behaviors Good for the } \\
\text { Environment }\end{array}$} & \multicolumn{2}{|c|}{$\begin{array}{l}\text { (Q3) Behaviors Whether } \\
\text { Respondents Wonder Are } \\
\text { Good or Bad }\end{array}$} & \multicolumn{2}{|c|}{$\begin{array}{l}\text { (Q4) Behaviors Generally } \\
\text { thought to Be Good While } \\
\text { Respondent Disagree }\end{array}$} \\
\hline & Word & Frequency & Word & Frequency & Word & Frequency \\
\hline 1 & Power saving & 18,193 & Nothing & 20,007 & Nothing & 29,110 \\
\hline 2 & Garbage separation & 18,100 & Garbage separation & 9315 & Don't know & 7280 \\
\hline 3 & Use & 8320 & No idea & 4638 & No idea & 3924 \\
\hline 4 & Not use & 6871 & Use & 3825 & Garbage separation & 3320 \\
\hline 5 & Ecobag & 6378 & Power saving & 3237 & Use & 1963 \\
\hline 6 & $\overline{\text { Nothing }}$ & 6129 & Recycle & 3037 & Recycle & 1627 \\
\hline 7 & Car & 5924 & $\overline{E c o b a g}$ & 1843 & Power saving & 1514 \\
\hline 8 & Recycle & 5437 & Don't know & 1788 & Nuclear power & 1398 \\
\hline 9 & Reduce garbage & 4389 & Garbage & 1528 & Eco bag & 1200 \\
\hline 10 & Water saving & 4108 & Car & 1470 & $\overline{\text { Eco car }}$ & 926 \\
\hline 11 & $\underline{\text { Turn off light }}$ & 3466 & PET bottle & 1325 & $\begin{array}{l}\text { Disposable wooden } \\
\text { chopsticks }\end{array}$ & 715 \\
\hline 12 & Garbage & 3189 & Purchase & 1272 & PET bottle & 678 \\
\hline 13 & Turn off frequently & 3043 & $\begin{array}{l}\text { Disposable wooden } \\
\text { chopsticks }\end{array}$ & 1126 & Idling stop & 677 \\
\hline 14 & Walk & 2516 & Nuclear power & 1105 & Electric car & 670 \\
\hline 15 & Bicycle & 2438 & Not use & 1002 & Not use & 658 \\
\hline 16 & Air conditioner & 2386 & Eco car & 920 & Think & 636 \\
\hline 17 & Wasting & 2198 & Replacement & 757 & Car & 621 \\
\hline 18 & Electricity & 2083 & Water & 720 & Hybrid car & 605 \\
\hline 19 & Purchase & 1818 & Electricity & 711 & Replacement & 602 \\
\hline 20 & Reduce use & 1627 & Eco products & 693 & Garbage & 591 \\
\hline
\end{tabular}

Bold \& underline: Power saving, Bold: Purchase, Italic: Garbage discharge, Italic \& underline: Resource saving (recycle), underline: Transport, wavy line: water saving fence-line: used in co-occurrence network analysis.

The results of co-occurrence network analysis indicate that major words co-occurring with the word "use (I use for the environment)" were "bicycle", "public transportation", "naturally derived", "forest thinning", "recycled products", "repair (long-term use)", "power saving", "refill type", and "natural soap (no detergent)". Meanwhile, the words co-occurring with "not use (I do not use for the environment)" were mainly "elevator", "escalator", "car", "detergent", "chemical fertilizer", "herbicide", and "paper cup".

Responses to Q3 including frequently appearing words and phrases are summarized in Table 7. Especially words related to "waste", "eco bag", "purchasing", and "car" were observed. Responses to Q4 indicated a tendency similar to Q3.

Characteristics by various socio-demographics are explained as follows, with details in Supplementary Materials. According to top 10 behaviors by age and gender for Q2 (Table S13), younger people provided "power saving". Men in their $30 \mathrm{~s}$ to $50 \mathrm{~s}$ provided behaviors relating to driving cars, whereas women in their $30 \mathrm{~s}$ to $50 \mathrm{~s}$ to garbage ("separation", "eco bag", and "recycle"). According to the top 10 behaviors by age and gender for Q3, the results show that people in their $10 \mathrm{~s}$ to $30 \mathrm{~s}$ doubted that "eco bag" and "power saving" were really good for the environment, even while those words were also inputted as good behaviors in Q2. They especially wondered if lights should be frequently turned on and off and whether there were more eco bags than actually needed. People in their $30 \mathrm{~s}$ to $40 \mathrm{~s}$ gave "garbage separation" as a top behavior. Men in their $30 \mathrm{~s}$ to $50 \mathrm{~s}$ doubted the benefits of "eco car" and "idling stop". Women in their $50 \mathrm{~s}$ to $60 \mathrm{~s}$ provided "wash for recycling"; they wondered whether 
washing recyclable garbage was really good for the environment, as this requires more water. Elderly men responded to behaviors in daily life as well as social issues such as "nuclear power", "solar power", and "carbon dioxide". There is a similar tendency observed between Q3 and Q4.

Table 7. Frequently appearing responses to question on behaviors that people were uncertain of as to whether they were good or bad for the environment $(\mathrm{Q} 3)$.

\begin{tabular}{cl}
\hline \multirow{2}{*}{ Waste } & $\begin{array}{l}\text { Separation, Combustion, Non-combustion, Different separation, Wash plastics, } \\
\text { Waste treatment, Increase of waste, Waste collection for recycle, Pick up trash, } \\
\text { Charge for waste collection }\end{array}$ \\
\hline \multirow{2}{*}{ Eco bag } & $\begin{array}{l}\text { Make, Utilize, Sale, Production, Mass production, Promotion, Distribution, } \\
\text { Excess of supply, Change }\end{array}$ \\
\hline \multirow{2}{*}{ Purchasing } & $\begin{array}{l}\text { Eco-marked products, PET bottle, Bulk purchase of eco bag or recycled products, } \\
\text { Ecological home electronics, Buy new eco products, Carbon offset, Buy goods } \\
\text { over internet, Eco car, Paper recycling }\end{array}$ \\
\hline Car & Electric car, Hybrid car, Gasoline-fueled car, Eco driving, Personal use \\
\hline Others & $\begin{array}{l}\text { Collecting milk cartons, } \mathrm{CO}_{2} \text { emission, All electrification, Open } 24 \text { h, Restart } \\
\text { nuclear power, Buy new even when not necessary }\end{array}$ \\
\hline
\end{tabular}

The top 10 behaviors by income for Q2 showed that people with lower income proposed garbage-related behaviors (Table S14). People whose income was under 2 million yen provided "no littering" or "throw away to trash box", which was rather a moralistic problem. People with higher income offered many words related to cars. This is partly because people with high income have higher car ownership rates. Public transportation was also seen in the top 10 words in higher income groups. Among the reasons for this is that people in urban areas tend to receive higher income on average and people whose houses are close to stations have the option to utilize public transportation (Figure S3). "Power saving" was seen in almost all income stages.

Among the top 10 behaviors by income for Q3, "replacement" and "purchase" were widely seen in groups with income of more than 4 million yen. Responses reflected that many considered whether the replacement of home appliances, cars, or still-usable things were harmful to the environment. "Eco car", "hybrid car", and "electric car" were also provided, meaning that people did not always consider the so-called eco car to be good for the environment.

As for regional differences, people who live in the northern parts of gave "garbage separation" and "power saving" as the top behaviors, whereas people who live in the southern region of Kansai mainly gave "power saving" and a few garbage-related words as top behaviors (Table S15). People in the Chubu area showed especially interesting responses; they provided car-related words as PEBs and garbage separation as a doubtful behavior. One possible reason for this finding is that the Chubu area includes mostly rural areas where cars are the main source of mobility. Another possible reason is that Nagoya City applies a very fragmented separation system for garbage collection. A similar pattern was observed in Q4 in each group; the explanation on Q4, hence, was omitted.

\subsubsection{Characteristics of the Five Clusters' Behavior Recognition}

The popular behaviors in each cluster of cluster analysis (five clusters) for Q2 to Q4 were also analyzed (Table 8). Although people in clusters 1 to 3 often answered the prompt at the word level, most people in clusters 4 and 5 were considered environmentally conscious as they expressed their thoughts and opinions using longer sentences. As such, feature expression analysis was conducted for clusters 4 and 5 (Table 9) as catching the major topic by only using word frequency analysis was difficult. 
Table 8. Top 10 frequently answered behaviors by 5 clusters; (Q2) Behaviors good for the environment;

(Q3) Behaviors uncertain of as to whether good or bad.

\begin{tabular}{|c|c|c|c|c|c|}
\hline (Q2) & & & & & \\
\hline & Cluster 1 & Cluster 2 & Cluster 3 & Cluster 4 & Cluster 5 \\
\hline 1 & Nothing & Not use & Nothing & Power saving & Reduce using \\
\hline 2 & Power saving & Use & Turn off light & Garbage separation & Reduce garbage \\
\hline 3 & Garbage separation & Garbage separation & Environment & Recycle & Raw garbage \\
\hline 4 & Eco bag & Air conditioner & No litter garbage & Energy saving & No purchase \\
\hline 5 & Turn off light & Turn off frequently & Train & Use & Purchase \\
\hline 6 & Turn off frequently & Unplug & Good & Eco bag & Use \\
\hline 7 & Water saving & Purchase & Not use & $\underline{\text { Car }}$ & Detergent \\
\hline 8 & Recycle & No purchase & Turn off frequently & Reduce garbage & Electricity \\
\hline 9 & Idling stop & Wasting & No littering & Eco & Wasting \\
\hline 10 & Air conditioner & Bicycle, Walk & Water saving & Solar power & Energy \\
\hline \multicolumn{6}{|l|}{ (Q3) } \\
\hline & Cluster 1 & Cluster 2 & Cluster 3 & Cluster 4 & Cluster 5 \\
\hline 1 & Nothing & Garbage separation & Garbage separation & Nothing & Use \\
\hline 2 & Garbage separation & Use & Power saving & Nuclear power & Good \\
\hline 3 & Power saving & Recycle & Eco bag & Water & Nuclear power \\
\hline 4 & Recycle & Eco bag & Eco car & Food & Water \\
\hline 5 & Eco bag & PET bottle & Idling stop & Can use & Environment \\
\hline 6 & Water saving & Replacement & Hybrid car & Raw garbage & Wonder good or not \\
\hline 7 & Idling stop & Eco car & Plastic bag & Don't know & Collection \\
\hline 8 & Air conditioner & Purchase & Air conditioner & Clean & All-electric house \\
\hline 9 & Plastic bag & Eco products & Recycle & Wash for recycling & Plastic \\
\hline 10 & Turn off light & Carry my-chopsticks & Carbon dioxide & No & Get dirty \\
\hline
\end{tabular}

Answers as regards power saving and garbage collection were widely seen as behaviors that are good for the environment in all clusters except for cluster 5 . In clusters 4 and 5 , the higher conscious groups, the frequently appeared behaviors were related to reducing garbage and saving resources. In particularly, cluster 5 provided "composting raw garbage" and "no purchase." In contrast, a lower attitude was seen in cluster 3 compared with other clusters; cluster 3 mainly gave "turn off light" and "no littering" as frequently appearing words.

As for Q3, "garbage separation", "eco bag", "recycle", and "power saving" were drawn in clusters 1 to 3, whereas "nuclear power" and "appropriate garbage separation or treatment" appeared in clusters 4 and 5. People in clusters 4 and 5 considered whether wiping off dirty dishes before washing them is better or not as this behavior could save water but increase the amount of garbage. They also wondered whether washing greasy plastics with food for recycling was beneficial as this requires more water and, thus, affects water treatment systems. These respondents already had basic environmental knowledge and seemed to require more precise information or data. 
Table 9. Top 10 feature expressions of answers by clusters 4 and 5 (5 clusters); (Q2) Behaviors good for the environment; (Q3) Behaviors uncertain of as to whether good or bad; (Q4) Behaviors generally thought to be good while respondent disagree.

\begin{tabular}{|c|c|c|c|c|c|}
\hline \multicolumn{2}{|c|}{ (Q2) Behaviors Good for the Environment } & \multicolumn{2}{|c|}{$\begin{array}{l}\text { (Q3) Behaviors Uncertain of as to } \\
\text { Whether Good or Bad }\end{array}$} & \multicolumn{2}{|c|}{$\begin{array}{l}\text { (Q4) Behaviors Generally thought to } \\
\text { Be Good While Respondent Disagree }\end{array}$} \\
\hline Cluster 4 & Cluster 5 & Cluster 4 & Cluster 5 & Cluster 4 & Cluster 5 \\
\hline Ecobag-Shopping & $\begin{array}{c}\text { Environment- } \\
\text { Consider (Select } \\
\text { products/service) }\end{array}$ & $\underbrace{\text { Clean-Wash }}_{\text {(Plastics) }}$ & $\begin{array}{l}\text { People-Bad } \\
\text { behavior for } \\
\text { environment }\end{array}$ & $\begin{array}{c}\text { PET } \\
\text { bottle-Recycling }\end{array}$ & $\begin{array}{c}\text { Eco } \\
\text { products-Think }\end{array}$ \\
\hline Resource-Reuse & $\begin{array}{l}\text { Raw garbage-Bury } \\
\text { (Yard/field) }\end{array}$ & $\begin{array}{c}\text { Garbage } \\
\text { separation-Discharge }\end{array}$ & $\begin{array}{c}\text { Garbage } \\
\text { separation-Wash }\end{array}$ & $\begin{array}{c}\text { Environment- } \\
\text { Not good (Various) }\end{array}$ & $\begin{array}{l}\text { Ecological home } \\
\text { appliances- } \\
\text { Replacement }\end{array}$ \\
\hline$\frac{\text { Electricity- }}{\text { Power saving }}$ & $\begin{array}{l}\text { Recycle-Be } \\
\text { conscious }\end{array}$ & $\begin{array}{c}\text { Garbage } \\
\text { separation-Different }\end{array}$ & $\begin{array}{l}\text { Get dirty-Wipe } \\
\text { off }\end{array}$ & Waste-Treatment & $\begin{array}{c}\text { Air-Keep clean } \\
\text { (Nuclear power, } \\
\text { Eco car) }\end{array}$ \\
\hline$\frac{\text { Cooling and heating- }}{\text { Temperature setting }}$ & $\begin{array}{c}\text { Reduce } \\
\text { garbage-Life }\end{array}$ & $\begin{array}{l}\text { Eco-Wonder } \\
\quad \text { (Various) }\end{array}$ & $\underline{\text { Car-Go outside }}$ & $\frac{\text { Plastic garbage- }}{\underline{\text { Wash }}}$ & $\frac{\frac{\text { Doubt using-Solar }}{\text { power, All-electric }}}{\frac{\text { house, Eco bag, }}{\text { washing machine) }}}$ \\
\hline $\begin{array}{l}\text { Water saving } \\
\text { Be conscious }\end{array}$ & Compost-Make & $\underbrace{\text { Dish Wash }}_{\text {(wipe off) }}$ & Plastic_-Wash & Troublesome-Take & $\begin{array}{c}\text { Nuclear } \\
\text { power-Make }\end{array}$ \\
\hline$\frac{\text { Power saving- }}{\underline{\text { Make effort }}}$ & Water-Not make & $\begin{array}{l}\text { Energy-Consumption } \\
\quad \text { (Recycle, PEB) }\end{array}$ & $\begin{array}{l}\text { Nuclear } \\
\text { power-Restart }\end{array}$ & $\begin{array}{l}\text { Wash for } \\
\text { recycling } \\
\text { Discharge }\end{array}$ & $\begin{array}{c}\text { Catchy } \\
\text { phrase-Do }\end{array}$ \\
\hline
\end{tabular}

Bold \& underline: Power saving, Bold: Purchase, Italic: Garbage discharge, Italic \& underline: Resource saving (recycle), underline: Transport, wavy line: water saving.

\section{Discussion}

\subsection{Comparison with Existing Surveys}

Similar prompts were put forward in existing surveys conducted by various organizations, specifically as positive questions. A comparison between those results and the present study's findings was shown in Figure 5. On one hand, 72.5\% answered "yes" when prompted with "I am concerned about environmental issues" in a positive sentence [12]. On the other hand, this present study showed that such positive respondents decreased to $41.4 \%$ when asked in a negative way. As regards consciousness in purchasing, $77.3 \%$ showed positive attitudes when asked in a positive way [13] but decreased to $58.9 \%$ when asked in a negative way. As regards displaying PEBs and inconveniences, about $80 \%$ people showed a positive attitude in a previous study [14] but the rate dropped to $35.5 \%$ when asked in a negative way. On individual influence on the environment, over $90 \%$ showed a positive attitude $[14,15]$ but the rate fell to about $65 \%$ when asked in a negative way. 


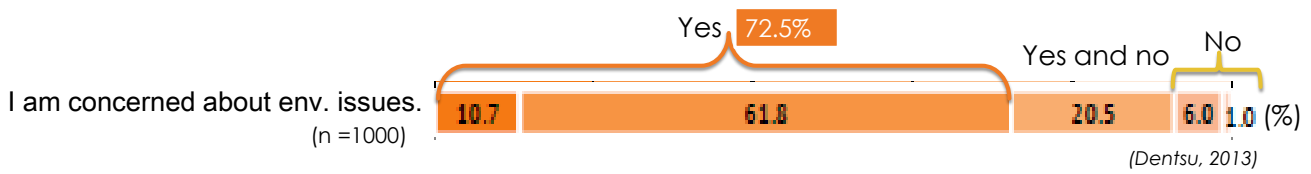

(a)

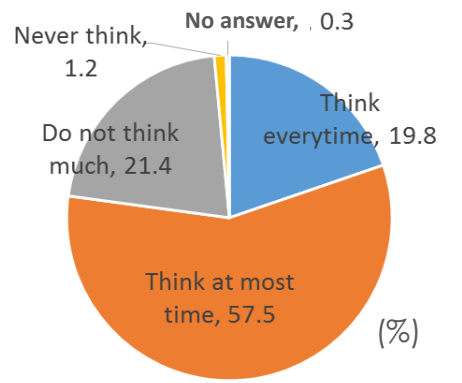

(Cainet Office, 2004)

I think about env. (e.g., energy, resources, and waste) in purchasing.

(b)

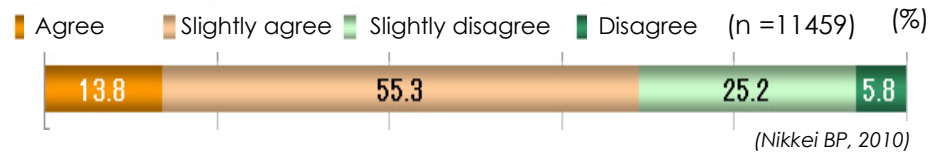

(c)

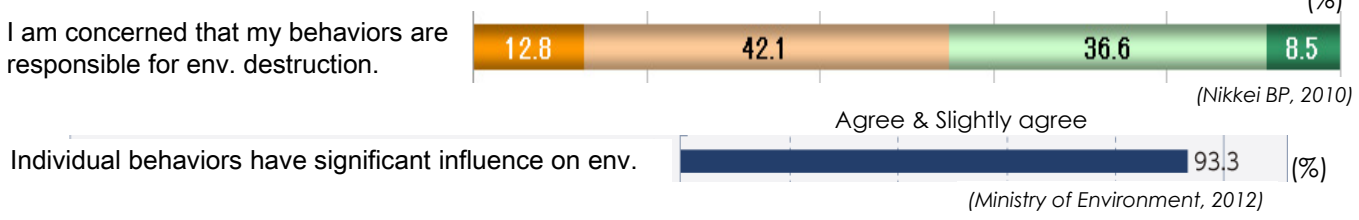

(d)

Figure 5. Responses to positive prompts: (a) Environmental consciousness; (b) Environmental awareness in purchasing; (c) PEB as inconvenient; (d) Personal responsibility.

Comparing with other surveys that employed positive questions, the rate of positive attitude for the environment in daily life was overall about $10 \%-20 \%$ lower on average. Such a result indicated that the survey with only positive prompts might have caused the higher environmental consciousness. However, the tendency was similar and people displayed positive attitudes in general.

\subsection{Socio-Demographics Effects and Psychological Elements of Environmental Awareness}

The relationship of socio-demographics and environmental consciousness were observed in this present study. From responses to 25 prompts, five psychological elements were extracted by factor analysis, and the specific differences in environmental attitude affected by demographic characteristics were determined.

First, differences among age and gender were observed. For example, women were found to be more environmentally conscious than men across the five factors. This finding is consistent with and strengthens the results of previous studies. Lee et al. and Aoyagi-Usui et al. reported that Japanese women were more likely to be positive than men in PEBs related to household affairs and consumer behaviors $[16,17]$. Barr also showed females were more likely to take waste prevention measures [18]. In the present study, women were more interested in waste-related behavior. Further, women often performed environmentally conscious actions based on custom rather than knowledge. Women also expressed difficulty in obtaining appropriate environmental information. In general, it was found that men had more knowledge on environmental issues than women but it did not indicate that men were more environmentally conscious or displayed PEBs more often. Although some women felt that they were lacking environmental information and were not sure of what was 
good or bad for the environment, they were aware of the environment in their daily lives. This is consistent with the previous research by Schahan and Holzer [19], who noted that "women were more environmentally concerned in those topical areas that refer to household behavior, whereas men knew more about environmental problems".

Further, older people were found to be more environmentally conscious than younger people as Ebreo et al. said in their report that positive correlation was shown between age and environmental attitude [20]. However, in the present study, respondents in their $20 \mathrm{~s}$ comprised the least conscious generation. Results of this present study also indicated that there was almost no difference in the types of consciousness according to age, but a large variance in consciousness among generations was found. In general, men in their $20 \mathrm{~s}$ and $30 \mathrm{~s}$ were the least environmentally conscious group, whereas elderly women were the most environmentally conscious group. As Barr said [18], older people showed more interest in waste separation, waste prevention, and recycling, according to text analysis.

Positive correlations between income and environmental consciousness for all five factors were also found in the present study. Hines et al. and Scott and Willtis reported that people with a higher income were more likely to engage in PEBs [21,22]. In the present study, the low attitude of people with income under 2 million yen and the high attitude of people with more than 15 million income were distinctive compared with the middle income group. Occupation was also found to have a certain effect on consciousness, especially awareness in daily life, self-reliance, as well as attainment of environmental information. However, there was an opposite tendency between single and two-person life. This might be because, when people live together, they become more conscious of living in a sustainable and harmonious way with other people and society including the environment.

This present study indicated a disparity between interest in environmental issues in general and awareness in daily activity. Certain respondents displayed a high score of factor for general interest in environment while simultaneously showing a low score for personal responsibility and awareness in everyday life. Certain people also agreed on the importance of environmental issues in general while, at the same time, performing PEBs only because others observe them to display these behaviors.

The factor of caring about the perceptions of others or society was extracted in this present study. Ando et al. reported that the Japanese subjective norm, which is a psychological element that considers what close people such as family members think, had a greater influence on Japanese in performing PEBs than it did for Germans. Other studies showed that Japanese people cared about other's points of view as part of their culture, and the effect of such attitude on their behaviors was larger than in other cultures $[16,23,24]$. Aoyagi-Usui et al. also concluded in their report that the structure of environmental values and factors encouraging environmental actions differ by country and type of actions [17]. Understanding the background culture and psychological characteristics of people to be able to approach them for involvement in PEBs has been seen significant.

\subsection{Respondents' Perceptions of PEBs}

Respondents were classified into five groups based on the factor scores of five factors: considerably low conscious group, slightly low conscious group, high-interest and low-information group, slightly high conscious group, and considerably high conscious group. More than half of the respondents were in the middle-level group and still have room for raising environmental awareness. This present study investigated the environmental consciousness level of the respondents. Concrete behaviors related to environmental issues in daily life were also provided by respondents. The connection between consciousness level and perceptions of behaviors was analyzed.

As regards PEBs, many respondents in the lower conscious group answered nothing (they do not know or cannot think of). This suggested that they were not aware of the environment in their daily lives. Among their answers, the frequent behaviors were turning off the light, turning down the air conditioning temperature, saving water, and appropriately disposing of garbage; their answers were easily actionable compared with other behaviors. Higher-interest and higher-conscious groups also included power saving behaviors and garbage separation in their responses, as well 
as behaviors related to resource saving such as using their own shopping bags, reducing garbage disposal, recycling, and limiting purchases; the latter behaviors require effort to some extent. Further, most conscious people identified behaviors that require more effort such as composting and selecting environmentally-friendly products and services. They did not mind time-consuming work and prioritized the environment over affluence and convenience. Hence, the content of concrete PEBs varied across different consciousness levels.

Most people gave examples of similar behaviors when asked about those they were doubtful or uncertain about in regards to what is good or bad for the environment: garbage separation, power saving, using eco bags, water saving, recycling, purchase for replacement, idling stops, and eco cars, among others. The most conscious people raised broader questions such as power generation and the so-called ecological products, which were issues beyond daily activities.

In this present study, words related to "garbage" were the most frequently provided. This is likely because garbage separation and collection systems are quite different depending on the municipality in Japan. Households in certain municipalities have to separate their garbage into more than 10 types, whereas those in other areas separate their garbage into only two or three types. Many people appear to wonder or doubt whether these measures are appropriate.

Overall, the same words and behaviors were very similar in their answers to Q2 (good behavior), Q3 (uncertain behavior), and Q4 (doubtful behavior). It was observed that many people were worried about actions that involved a trade-off relationship from diversified standpoints. For example, they wondered if it is really good to use ample water to wash plastic garbage prior to recycling even though they regard recycling as a good thing. Thus, people with higher consciousness sometimes worried more in certain circumstances. Environmental issues include multifaceted aspects. People do not have enough information and ability to compare or evaluate the entire environmental load throughout the life cycle as a consequence of their behaviors. Thus, it is important to support people to develop life cycle thinking so that they can find the best practices for the environment. This would require providing information and datasets to the public, which are easy to access, easy to understand and customized to people's different levels of consciousness and specific needs.

\section{Conclusions}

A nationwide online survey on environmental consciousness and perceptions of PEBs in daily life was conducted in Japan. This survey consisted of negative prompts to remove the "yes-bias" tendency of positively asked prompts. However, PEBs are considered complex and encompass different levels of issues. Hence, dividing attitudes into positive or negative positions was a difficult task. However, the present study applied the reversely expressed prompts and explained them as negative prompts in an attempt to focus on the expression styles used in existing surveys and, thereby, examine the effect of positively expressed prompts. Free-answered prompts that asked three kinds of behaviors were also included in the survey to investigate the connection of the level of consciousness and concrete behaviors in daily life. These three kinds of behaviors were behaviors people consider as good for the environment, those that they were uncertain as to whether they were good or bad for the environment, and those that are generally good for the environment but they did not agree on. A large sample of more than 70,000 people was collected to represent the situation in Japan.

The level of environmental consciousness was lower in the survey with negative prompts than in other surveys with positive prompts. However, people's positive attitudes to the environment were observed overall. The demographic effects on the responses were also investigated.

By factor analysis with responses, five psychological factors were extracted as the explanatory factors for environmental attitudes. The results of the analysis suggested that gender, age, income, and occupation had a strong relationship with environmental consciousness. The number of household members also had an effect to some extent. The area of residence of respondents had almost no influence.

Based on factor analysis, respondents were classified into five groups by cluster analysis. More than half of the respondents were included in the middle two groups, which had a slightly higher or 
lower consciousness of environmental impacts. Other groups were considerably low or high conscious groups and high-interest and low-information groups. The comparison between free-answers for concrete daily behaviors among five different environmentally conscious groups showed that there were several phases in perception of PEBs according to the level of consciousness, whereas almost all groups answered similar behaviors as uncertain behaviors. The most conscious group showed distinctive behaviors for all answers.

The word frequency analysis and co-occurrence network analyses for responses of free-answer prompts showed that the similar common behaviors were highly ranked for all prompts. Differences were observed depending on gender, age, income, and consciousness level as mentioned above. People worried about actions that involved a trade-off relationship when they considered various aspects. It will be important to provide people access to various information sources and data sets to support their decisions in selecting the appropriate behavior as well as to provide a basis to build people's environmental consciousness for daily living.

Supplementary Materials: Supplementary material can be found at www.mdpi.com/2071-1050/8/1/24/s1.

Acknowledgments: This research was supported by Environment Research and Technology Development Fund (Development of LCA Database and Educational Materials about Daily Behaviors aiming for Common Platform Provision) of the Ministry of the Environment, Japan.

Author Contributions: Ai Hiramatsu and Kiyo Kurisu conceived and designed the research; Ai Hiramatsu performed the research survey and analyzed the data; Kiyo Kurisu advised on data analysis; Ai Hiramatsu wrote the paper. Kiyo Kurisu and Keisuke Hanaki reviewed and commented on the paper.

Conflicts of Interest: The authors declare no conflict of interest.

\section{References}

1. Harju-Autti, P.; Kokkinen, E. A Novel Environmental Awareness Index Measured Cross-Nationally for Fifty Seven Countries. Univers. J. Environ. Res. Technol. 2014, 4, 178-198.

2. Kollmuss, A.; Agyeman, J. Mind the Gap: Why do people act environmentally and what are the barriers to pro-environmental behavior? Environ. Educ. Res. 2002, 8, 239-260. [CrossRef]

3. Giuseppe, I. The Power of Survey Design: A User's Guide for Managing Surveys, Interpreting Results, and Influencing Respondents; The International Bank for Reconstruction and Development: Washington, DC, USA, 2006; pp. 44-46.

4. Berg, I.A.; Rapaport, G.M. Response bias in an unstructured questionnaire. J. Psychol. 1954, 38, 475-481. [CrossRef]

5. Chao, L.; Lam, S.P. Measuring Responsible Environmental Behavior: Self-Reported and Other-Reported Measures and Their Differences in Testing a Behavioral Model. Enviorn. Behav. 2011, 43, 53-71. [CrossRef]

6. Kaiser, F.G.; Ranney, M.; Hartig, T.; Bowler, P.A. Ecological behavior, environmental attitude, and feelings of responsibility or the environment. Eur. Psychol. 1999, 4, 59-74. [CrossRef]

7. Milfont, T.L. The effects of social desirability on self-reported environmental attitudes and ecological behaviour. Environmentalist 2009, 29, 263-269. [CrossRef]

8. Ajzen, I. The theory of planned behavior. Organ. Decis. Hum. Decis. Process 1991, 50, 179-211. [CrossRef]

9. Stern, P.C. Information, incentives, and proenvironmental consumer behavior. J. Consum. Policy 1999, 22, 461-478. [CrossRef]

10. Stern, P.C. New Environmental Theories: Toward a Coherent Theory of Environmentally Significant Behavior. J. Soc. Issues 2000, 56, 407-424. [CrossRef]

11. Statistics Bureau. Portal Site of Official Statistics of Japan: E-Stat. Available online: http://www.e-stat. go.jp/SG1/estat/List.do?bid=000001034991\&cycode $=0$ (accessed on 16 November 2015).

12. Dentsu. News Release of the Report of Dentsu Green Consumer Survey 2013. Available online: http:/ /www. dentsu.co.jp/news/release/2013/pdf/2013048-0411.pdf (accessed on 28 September 2015). (In Japanese)

13. Cabinet Office. National Life Monitor Survey Results (Summary) - Survey on Environmental Consciousness 2004. Available online: http://www5.cao.go.jp/seikatsu/monitor/pdf/h1603monitor.pdf (accessed on 28 September 2015). (In Japanese). 
14. Nikkei BP Consulting, Inc. Trend Survey: Eco Consciousness/Behavior Research; Nikkei BP Consulting, Inc.: Tokyo, Japan, 2010. (In Japanese)

15. Ministry of Environment. Summary of Public Opinion Survey on Environmental Issues, 2012. Available online: https: / / www.env.go.jp/press / file_view.php?serial=20437\&hou_id=15543 (accessed on 28 September 2015). (In Japanese)

16. Lee, H.; Kurisu, K.; Hanaki, K. Influential Factors on Pro-Environmental Behaviors-A Case Study in Tokyo and Seoul. Low Carbon Econ. 2013, 4, 104-116. [CrossRef]

17. Aoyagi-Usui, M.; Vinken, H.; Kuribayashi, A. Pro-Environmental Attitude and Behaviors: An International Comparison. Hum. Ecol. Rev. 2003, 10, 23-31.

18. Barr, S. Strategies for sustainability: Citizens and responsible environmental behavior. Area 2003, 35, $227-240$. [CrossRef]

19. Schahn, J.; Holzer, E. Studies of individual environmental concern: The role of knowledge, gender, and background variables. Environ. Behav. 1990, 22, 767-786. [CrossRef]

20. Ebreo, A.; Hershey, J.; Vining, J. Reducing solid waste: Linking recycling to environmentally responsible consumerism. Environ. Behav. 1999, 31, 107-135. [CrossRef]

21. Hines, J.M.; Hungerford, H.R.; Tomera, A.N. Analysis and Synthesis of Research on Responsible Environmental Behavior: A Meta-Analysis. J. Environ. Educ. 1987, 18, 1-8. [CrossRef]

22. Scott, D.; Willtis, F.K. Environmental attitudes and behavior: A Pennsylvania survey. Environ. Behav. 1994, 26, 239-260. [CrossRef]

23. Abrams, D.; Ando, K.; Hinkle, S. Psychological attachment to the group: Cross-cultural differences in organizational identifications and subjective norms as predictors of workers' turnover intentions. Personal. Soc. Psychol. Bull. 1998, 24, 1027-1039. [CrossRef]

24. Ando, K.; Ohnuma, S.; Chang, E.C. Comparing normative influences as determinants of environmentally-conscious behavior between the U.S. and Japan. Asian J. Soc. Psychol. 2007, 10, 171-178. [CrossRef]

(C) 2015 by the authors; licensee MDPI, Basel, Switzerland. This article is an open access article distributed under the terms and conditions of the Creative Commons by Attribution (CC-BY) license (http://creativecommons.org/licenses/by/4.0/). 